\title{
BMJ Open Predictors of relapse in MOG antibody associated disease: a cohort study
}

\author{
Saif Huda (D) , ${ }^{1}$ Daniel Whittam, ${ }^{1,2}$ Richard Jackson, ${ }^{3}$ Venkatraman Karthikeayan, ${ }^{1}$ \\ Patricia Kelly, ${ }^{1}$ Sam Linaker, ${ }^{1}$ Kerry Mutch, ${ }^{1}$ Rachel Kneen, ${ }^{4,5}$ Mark Woodhall, ${ }^{6}$ \\ Katy Murray, ${ }^{7}$ David Hunt, ${ }^{7,8}$ Patrick Waters, ${ }^{6}$ Anu Jacob ${ }^{1,9}$
}

To cite: Huda S, Whittam D, Jackson $\mathrm{R}$, et al. Predictors of relapse in MOG antibody associated disease: a cohort study. BMJ Open 2021;11:e055392. doi:10.1136/ bmjopen-2021-055392

- Prepublication history and additional supplemental material for this paper are available online. To view these files, please visit the journal online (http://dx.doi.org/10.1136/ bmjopen-2021-055392).

Received 14 July 2021 Accepted 02 November 2021
Check for updates

(C) Author(s) (or their employer(s)) 2021. Re-use permitted under CC BY-NC. No commercial re-use. See rights and permissions. Published by BMJ.

For numbered affiliations see end of article.

Correspondence to Dr Saif Huda; shuda@nhs.net

\section{ABSTRACT}

Objective To identify factors predictive of relapse risk and disability in myelin oligodendrocyte glycoprotein associated disease (MOGAD).

Setting Patients were seen by the neuromyelitis optica spectrum disorders (NMOSD) service in Liverpool, UK, a national referral centre for adult patients with MOGAD, NMOSD and related conditions.

Participants Patients with MOGAD $=76$ from England, Northern Ireland and Scotland were included in this cohort study.

Results Relapsing disease was observed in 55\% (42/76) of cases. Steroid treatment $\geq 1$ month $(0 \mathrm{R} 0.2,95 \% \mathrm{Cl} 0.05$ to $0.80 ; p=0.022)$, transverse myelitis (TM) at first attack (OR $0.03,95 \% \mathrm{Cl} 0.004$ to $0.23 ; \mathrm{p}=0.001$ ) and male sex (OR $0.16,95 \% \mathrm{Cl} 0.04$ to $0.68 ; \mathrm{p}=0.014$ ) were associated with monophasic disease (area under the curve $=0.85$ ). Male sex (HR 0.46, 95\% Cl 0.24 to 0.89; $p=0.011$ ) and TM at disease onset ( $\mathrm{HR} 0.42,95 \% \mathrm{Cl} 0.22$ to $0.82 ; \mathrm{p}=0.011$ ) were also associated with an increased latency to first relapse. $45 \%$ (32/71) of patients became MOG-antibody negative and in relapsing patients negative seroconversion was associated with a lower relapse risk (relative risk 0.11 $95 \% \mathrm{Cl} 0.05$ to $0.26 ; p<0.001)$. No specific factors were predictive of visual or overall disability.

Conclusions Male patients with spinal cord involvement at disease onset have a lower risk of relapsing disease and longer latency to first relapse. Steroid treatment for at least 1 month at first attack was also associated with a monophasic disease course. MOG-antibody negative seroconversion was associated with a lower risk of relapse and may help inform treatment decisions and duration.

\section{INTRODUCTION}

Myelin oligodendrocyte glycoprotein antibody associated disease (MOGAD) is associated with central nervous system (CNS) inflammation, typically acute disseminated encephalomyelitis, optic neuritis $(\mathrm{ON})$, transverse myelitis (TM) and brainstem inflammation. ${ }^{1-7}$ In retrospective studies, a relapsing disease course has been reported in 27\%-80\% of patients, which may over-report the proportion of relapsing patients by virtue of differential follow-up of monophasic versus relapsing patients. ${ }^{1-5}$ Indeed in two studies using incident cohorts, rates of relapsing disease were

\section{Strengths and limitations of this study}

- This UK cohort study of myelin oligodendrocyte glycoprotein associated disease (MOGAD) included 76 patients from England, Northern Ireland and Scotland.

- Prognostic factors associated with relapsing disease and time to first relapse were assessed using univariable and multivariable modelling.

- The longitudinal impact of MOG antibody disappearance on relapse risk was analysed using a Poisson regression model.

- A limitation of this study was the shorter duration of follow-up in monophasic patients with MOGAD.

at the lower end $(27 \%-36 \%) .{ }^{12}$ Although MOGAD is associated with a better prognosis compared with neuromyelitis optica spectrum disorder (NMOSD), persistent visual, motor or sphincter disturbances have been reported. ${ }^{17}$ These studies collectively support the presence of a subgroup of patients with MOGAD with lower risk of relapse and minimal if any long-term disability. This has understandably led to equipoise among international experts on when to introduce chronic immunotherapy and the duration of treatment. ${ }^{8}$ Identifying prognostic factors for risk of (1) early relapse, (2) any relapse and (3) permanent disability will help individualise MOGAD treatment.

\section{Cohort description}

Study design

All patients were seen by the NMOSD UK service at the Walton Centre NHS Foundation Trust in Liverpool, UK, a national referral centre for adult patients with NMOSD, MOGAD and other non-multiple sclerosis atypical CNS inflammatory/demyelinating syndromes. Patients from England, Northern Ireland and Scotland were included.

Between January 2010 and January 2020 , patients with an acute demyelinating syndrome, at least one serum MOG-IgG1 positive assay result, and a minimum of 12 
months follow-up were included. Serum MOG-IgG1 Abs were detected using a live cell-based assay employing fulllength human MOG ( $\alpha 1$ isoform; Oxford Autoimmune Neurology Group) ${ }^{9}$

Demographic, clinical details of attacks, cerebrospinal fluid and MRI results, treatment and longitudinal MOG-Ab results were collected. Childhood onset was defined as disease onset at age $\leq 16$ years. Patients were considered 'monophasic' if no relapses were observed after the first clinical attack for the duration of follow-up (at least 12 months) and were compared with relapsing patients with MOGAD. Patients where the diagnosis was made shortly after onset ( $<6$ months) and prior to relapse were designated as 'incident' cases. The following outcomes were examined: (1) relapse at any time, (2) visual acuity $\leq 6 / 36$ (one or both eyes at last follow-up), (3) time between first and second attack and (4) impact of MOG-Ab serostatus on relapse frequency.

\section{Statistical analysis}

Continuous covariates are summarised as median (IQR) unless otherwise stated with categorical covariates summarised as frequencies with associated percentages. For comparisons of covariates across groups, Fisher's exact tests and Mann-Whitney U tests were applied for categorical and continuous data, respectively.

To evaluate the impact of covariates on each endpoint, univariable and multivariable modelling were applied. Multivariable models for binary endpoints were constructed using a generalised linear model assuming a binomial distribution and a logistic link function and using a forward stepwise approach. Model evaluations were performed using Akaike Information Criterion. Model performance were assessed by comparing the linear predictors against the model outcome using receiver operating curve (ROC) and area under the curve (AUC). Model results are presented in terms of ORs with associated 95\% CIs. For the time-to-event outcome, estimates of the probability of relapse were obtained using the Kaplan-Meier method. Univariable and multivariable analyses were performed using Cox proportional hazards models with an equivalent procedure used to evaluate univariate models and construct multivariable models. Results are presented in terms of HRs with 95\% CIs. The longitudinal impact of MOG-Ab negativity on patient relapse was investigated using a random effects Poisson regression model. Here, MOG-Ab negativity was included as a fixed effect and the time included in the model as a (log) offset. Patient identifier was included as a random effect. Results are presented as relative and absolute risk for observing a relapse with $95 \%$ CIs. A threshold of $\mathrm{p}<0.05$ was applied for statistical significance. All analyses were performed using R (V.3).

\section{Patient and public involvement}

Clinical data from patients were included in this study. The development of the research question was driven by our patient's uncertainty over future relapse risk following a first presentation of MOGAD. The patients and public were not explicitly involved the design or conduct of this study. Results will be disseminated at the NMOSD UK patient day where patients, relatives and caregivers will be in attendance.

\section{RESULTS}

\section{Demographic features}

We identified 76 patients with MOGAD with a median onset age of 27 (IQR 19-45), 54\% were female and 17\% had disease onset in childhood (age $\leq 16$ years). The geographic distribution of patients is shown in online supplemental figure 1. In total, 42 relapsing patients (total no of relapses $=140$ ) and 34 monophasic patients were identified. The median time from first clinical attack to diagnosis in the incident cohort $(\mathrm{n}=38)$ was 1 month (IQR 0-2 months). The clinical profile of patients and respective univariable analyses is presented in table 1 and online supplemental table 1.

Overall, there was a slight female predominance (54\%) and although the proportion of male patients did not reach statistical significance in the univariable analysis, male sex was associated with a lower overall risk of relapsing disease (OR $0.1695 \%$ CI 0.04 to $0.68 ; \mathrm{p}=0.014$ ) and time to first relapse (HR $0.4695 \%$ CI 0.24 to 0.89 ; $\mathrm{p}=0.011$ ) in multivariable analyses (figure 1, online supplemental table 2). The majority of patients (93\%) were white; there were no racial differences between the groups. The median age of relapsing patients was lower than monophasic patients (26 (16-40) versus 37 (27-51) years; $\mathrm{p}=0.001)$. Development of MOGAD after the age of 16 years was associated with a lower risk of relapsing disease in the multivariable analysis (OR 12.54 (1.81 to 87.17; $\mathrm{p}=0.011$ )), but $12 / 13$ children had relapses, suggesting a bias towards follow-up of children into adulthood with relapsing disease.

\section{Clinical course}

Relapsing disease was observed in $18 \%$ of incident cases and $55 \%$ of the total cohort with a median time to first relapse of 11.5 (IQR 3-46) months. A survival analysis for time to first relapse between the incident and total cohort is presented in online supplemental figure 2. The most common first clinical presentations were ON; $61 \%$, TM; $42 \%$ and bilateral ON (36\%, table 1). TM and longitudinally extensive transverse myelitis (LETM) were more frequently part of the first clinical attack in monophasic patients $(62 \%$ vs $26 \%, \mathrm{p}=0.002 \%$ and $41 \%$ vs $17 \%$; $\mathrm{p}=0.022$, respectively). In multivariable analysis, TM with a first attack was associated with a lower overall risk of relapse (OR 0.03 , 95\% CI 0.004 to 0.23 ; $\mathrm{p}=0.001$ ) and a longer time to first relapse (HR $0.4295 \%$ CI 0.22 to $0.82 ; \mathrm{p}=0.011$; figure 2 and online supplemental table 2). Importantly although median follow-up duration was longer in relapsing as compared with monophasic patients (107 (44-162) vs $33.5(20-56)$ months; $\mathrm{p}<0.001))$, the median follow-up times of these groups of patients with TM specifically were similar (35 (26-62) vs 55 (43-113); 
Table 1 Univariate analysis of relapsing and monophasic patients with myelin oligodendrocyte glycoprotein antibody associated disease

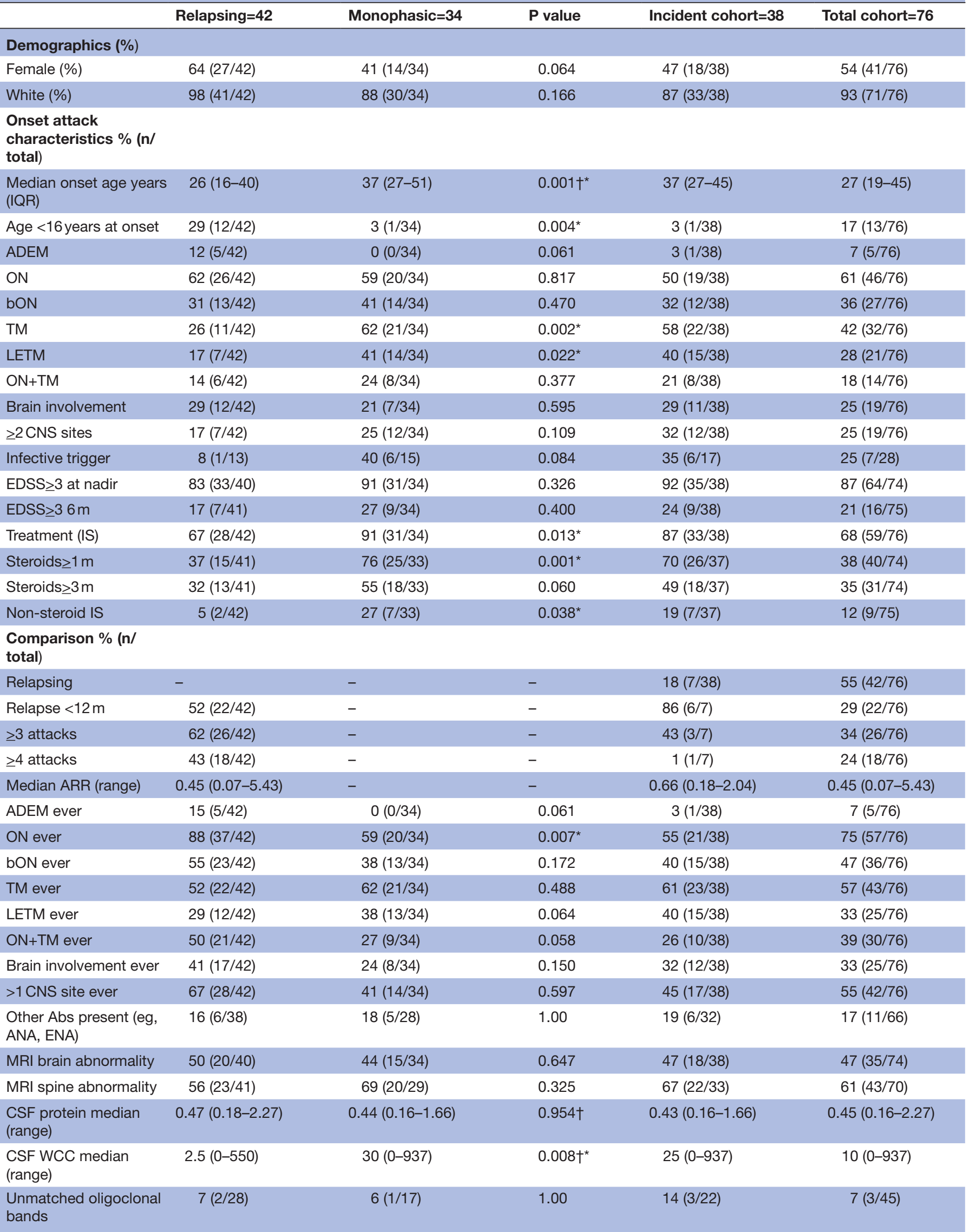


Table 1 Continued

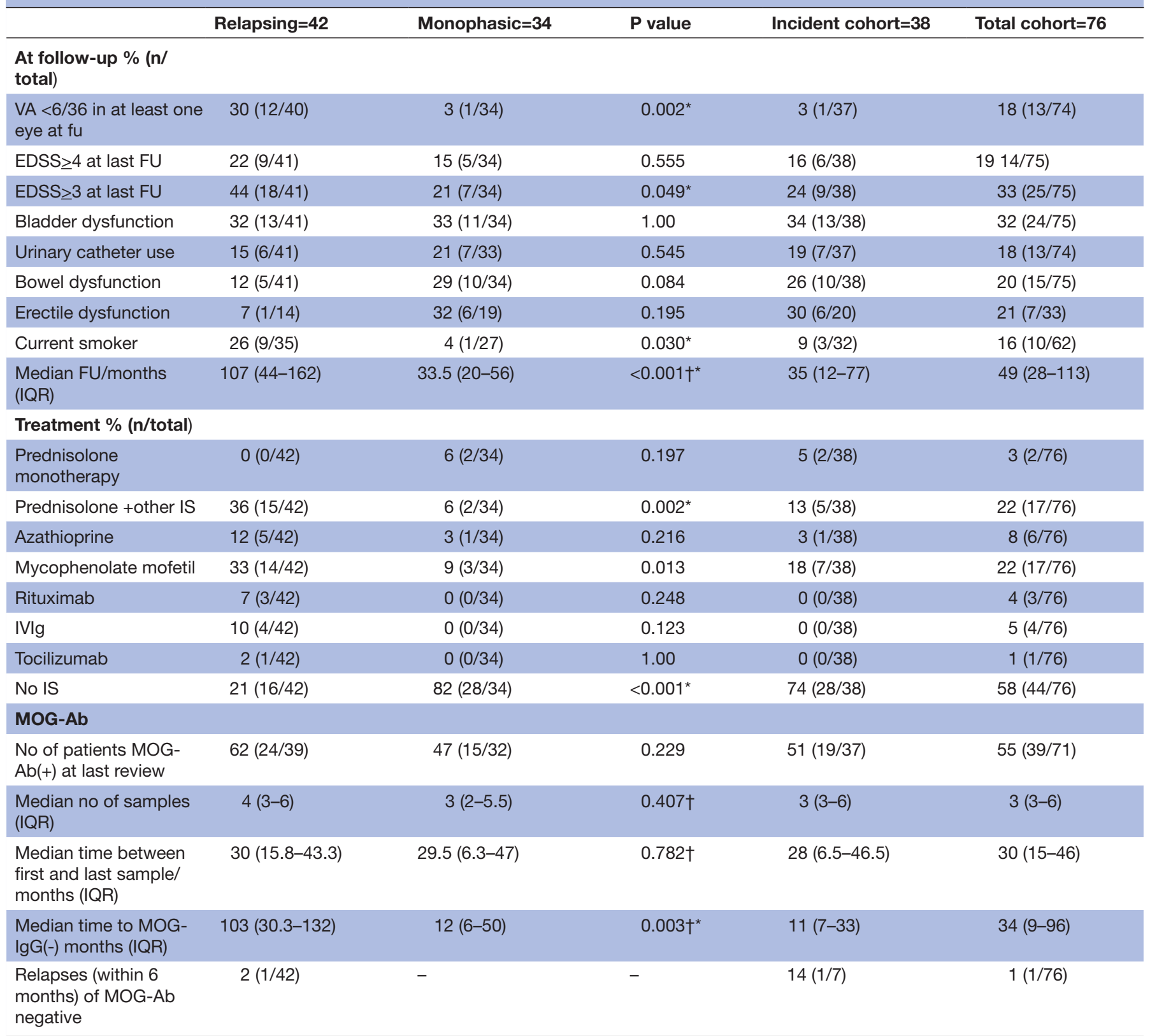

${ }^{*} \mathrm{P}<0.05$.

†Mann-Whitney U test.

Abs, antibodies; ADEM, acute disseminated encephalomyelitis; ANA, antinuclear antibody; ARR, annualised relapse rate; bON, bilateral ON; CNS, central nervous system; CSF, cerebrospinal fluid; EDSS, expanded disability status score; ENA, extractable nuclear antigen ; FU, follow-up; IS, immunosuppression; IVIg, intravenous immunoglobulin; LETM, longitudinally extensive TM; ON, optic neuritis; TM, transverse myelitis; VA, visual acuity; WCC, White Cell Count.

$\mathrm{p}=0.11$ ). In addition, there was no difference in use of steroids $>1 \mathrm{~m}$ in those patients presenting with or without $\mathrm{TM}(59 \%$ vs $50 \%$; $\mathrm{p}=0.485)$.

Simultaneous ON+TM at any point was associated with a greater risk of relapsing disease (OR 12.54 (1.81 to 87.17 ); $\mathrm{p}=0.011$ ), but follow-up duration was shorter in these patients with monophasic disease $(\mathrm{p}=0.018)$. The proportion of patients presenting with bilateral $\mathrm{ON}$ and multi-CNS site involvement were similar between relapsing and monophasic groups. Three patients presented with encephalitis and seizures, all of whom had a relapsing disease course. Expanded disability status score (EDSS) at nadir and 6 months after first attack was similar in monophasic and relapsing patients. Preceding infective symptoms were more frequent in monophasic patients, but the results did not reach statistical significance $(40 \%$ vs $8 \% ; \mathrm{p}=0.084)$.

Overall, more than 2 and 3 attacks were observed in $34 \%$ $(26 / 76)$ and $24 \%(18 / 76)$ of patients, respectively. Only $18 \%$ of incident cases relapsed and $86 \%$ of first relapses occurred within 12 months of the first attack. Follow-up duration was shorter in the incident cohort as reflected 


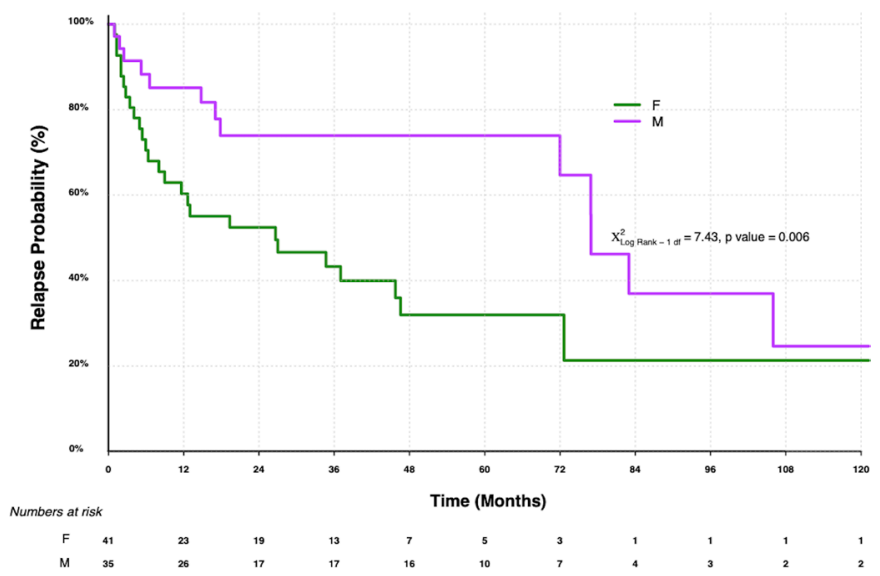

Figure 1 Kaplan-Meier analysis of relapse probability and sex.

in the higher annualised relapse rate (ARR) in these patients as compared with the total cohort (0.66 (0.18$2.04)$ vs $0.45(0.07-5.43))$. Smoking was more frequently noted at follow-up in relapsing patients $(26 \%$ vs $4 \%$; $\mathrm{p}=0.030$ ) but median ARR in smokers was similar to nonsmokers in the incident patients with MOGAD ( $\mathrm{p}=0.533)$. The most common CNS sites involved in attacks were the optic nerve $(57 / 76 ; 75 \%)$, spinal cord $(43 / 76 ; 57 \%)$ or simultaneous involvement of both these sites $(30 / 76$; $39 \%$ ). The site of CNS involvement was similar between relapsing and monophasic groups with the exception of ON, which was more common in relapsing patients $(88 \%$ vs $59 \% ; \mathrm{p}=0.007)$.

\section{Paraclinical tests}

In the total cohort, MRI abnormalities in brain and spine were observed in $47 \%$ and $61 \%$ of cases, respectively. The frequency of abnormalities on MRI brain $(p=0.647)$ and spinal cord $(\mathrm{p}=0.325)$ were similar between relapsing and monophasic patients. CSF white cell count was higher in monophasic patients $(\mathrm{p}=0.008)$. Unmatched oligoclonal bands were seen in only $3 / 45(7 \%)$ cases tested. Nonorgan specific autoantibodies (eg, antinuclear antigen, extractable nuclear antigen) were present in $16 \%$ of

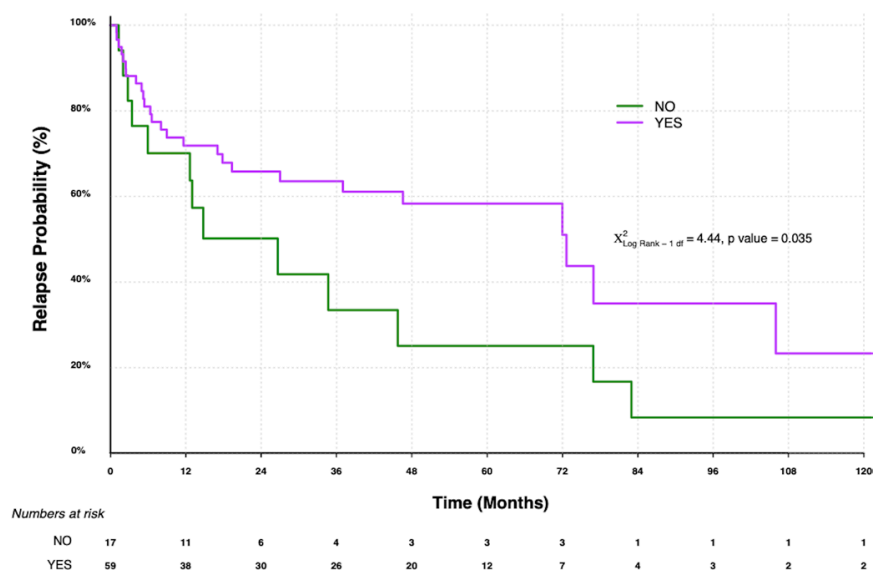

Figure 2 Kaplan-Meier analysis of relapse probability and transverse myelitis. relapsing and $18 \%$ and monophasic patients. None of these variables maintained a significant association in multivariable analysis.

\section{Treatment}

Overall, $38 \%(40 / 74)$ of patients received steroid treatment for $\geq 1$ month and $12 \%(9 / 75)$ were commenced on non-steroid immunosuppression (IS) following the onset clinical attack. Both steroid treatment for $\geq 1$ month (76\% vs $37 \% ; p=0.001)$ and non-steroid IS $(27 \%$ vs $5 \%$; $\mathrm{p}=0.038)$ were associated with monophasic disease. In multivariable analysis, treatment of the first attack with steroids $>1$ month was associated with a lower overall relapse risk (OR $0.2,95 \%$ CI 0.05 to $0.80 ; \mathrm{p}=0.022$, online supplemental table 2). In keeping with current UK practice, steroids $>1 \mathrm{~m}$ were more frequently used in incident as compared with non-incident patients $(70 \%$ vs $39 \%)$. Overall, 32/76 (42\%) of patients with MOGAD received long term IS, and of these patients 26 (81\%) had relapsing disease. In order of frequency, the most commonly used non-steroid immunosuppressants were mycophenolate mofetil $(22 \%)$ and azathioprine $(8 \%)$. Intravenous immunoglobulin (IVIg) (5\%), rituximab (4\%) and tocilizumab (1\%) were used as second-line and third-line therapies. In $22 \%$ of patients, maintenance prednisolone (5-15 mg/day) was combined with non-steroid IS.

An evaluation of the multivariable model to describe monophasic patients was performed using ROC analysis with the following factors-age $>16$ years, male sex, TM at onset, steroids $>1$ month. Using the linear predictor from the fitted model, an AUC of 0.92 was achieved. However, in view of the observer bias relating to age at disease onset, this variable was removed and a high AUC of 0.85 was maintained.

\section{Long-term outcome}

Poor visual outcome defined by a visual acuity of $\leq 6 / 36$ in at least one eye at last review was observed in $18 \%$ $(13 / 74)$ of patients after a median of 13.5 years follow-up (online supplemental table 1). Of those with poor visual outcome, $85 \%(11 / 13)$ had an EDSS $\geq 3 \%$ and $39 \%$ (5/13) had an EDSS $\geq 4$. Permanent visual disability (VA) was more common in relapsing MOGAD (30\% vs $3 \%$; $\mathrm{p}=0.002)$ and median follow-up duration in these patients was longer (median 161 vs 43 months; $\mathrm{p}<0.0001$ ). Interestingly patients presenting with TM or LETM at first attack were less likely to develop optic nerve involvement $(53 \%$ vs $91 \%$; $\mathrm{p}=0.0003 \%$ and $38 \%$ vs $89 \%$; $=0.0001$, respectively) and had a better visual prognosis (49\% vs $8 \%$; $\mathrm{p}=0.006 \%$ and $33 \%$ vs $0 \%$; $=0.015$, respectively, online supplemental table 1). In the multivariable analysis, TM with the first MOGAD clinical attack was associated with a favourable visual prognosis (OR 0.09 (0.01 to 0.70 ); $\mathrm{p}=0.022$, online supplemental table 2). To determine whether this simply reflected less optic nerve involvement in patients with TM at onset we analysed onset TM patients with subsequent $\mathrm{ON}$ attacks $(17 / 32 ; 53 \%)$ and the remaining patients that developed ON only after first 
attack $(12 / 30 ; 40 \%)$. Although the results were not significant, there was a trend towards better visual outcome in patients that presented with TM and had subsequent optic nerve involvement ( $6 \%$ vs $36 \%$; $\mathrm{p}=0.06$ ).

Patients with relapsing disease (92\% vs $46 \%$; $\mathrm{p}=0.002)$, $>3$ relapses $(83 \%$ vs $29 \%$; $\mathrm{p}=0.002)$ or a history of bilateral ON (77\% vs $43 \%$; $p=0.033)$ had worse visual outcomes but these factors and others (MOG-Ab seronegative status and long term IS) did not maintain a significant association in multivariable analysis. A visual acuity $\leq 6 / 36$ in at least one eye at last review was more frequently observed with childhood onset MOGAD (46\% vs 12\%; $\mathrm{p}=0.008$ ). As with findings related to higher relapse rate, this observation likely relates to preferential follow-up of children with more severe disease.

An EDSS $>3$ was recorded in 33\% (25/76) of patients at last review after a median of 6.6 years follow-up. Follow-up duration was longer in patients with an EDSS $>3$ (median 79 vs 44 months; $p=0.004$ ). Approximately a third of relapsing and monophasic patients had bladder dysfunction at last review. Rates of bowel and erectile dysfunction were not significantly different between relapsing and monophasic patients. Patients who received IVIg had worse EDSS (and visual) scores at follow-up due to refractory relapsing clinical disease with severe disability prior to treatment commencement. No clinical feature was associated with overall disability (EDSS $>3$ ) in multivariable analysis.

\section{MOG-Ab serostatus}

In total, 71 patients had more than 1 serum sample for MOG-Ab testing (table 1 ). The median number of samples in relapsing and monophasic groups (4 (IQR: 3-6) vs 3 (IQR: 2-5.5); $\mathrm{p}=0.407$ ) and patients with and without persistent MOG-Abs (3 (IQR: 3-6) vs 4 (IQR: $3-6) ; p=0.563$ ) were similar (online supplemental tables 1 and 2). The median time between first and last sampling (30 (15.8-43.3) vs 29.5 (21-53.8) months; $\mathrm{p}=0.782$ ) was also similar.

Persistent MOG-Ab detection was observed in 55\% $(39 / 71)$ of patients. In 2 patients MOG-Ab serostatus was initially negative and then became positive. In 5 patients, a fluctuating MOG-Ab serostatus was noted-following a positive MOG-Ab result 3 patients became transiently negative and then persistently positive and 2 patients became negative, positive and then persistently negative. The median time to negative MOG-Ab serostatus was 34 (9-96) months and as expected was shorter in the incident cohort (11 (7-33) months). The time interval was also shorter in the monophasic as compared with relapsing patient group (12 (6-50) vs 103 (30.3-132) months; $\mathrm{p}=0.003$ ). Relapse within 6 months of a negative MOG-Ab assay was recorded in only 1 patient. Two further patients had a relapse after a negative result, but MOG-Ab testing was done more than 6 months prior to attack and undetected MOG-Ab positive seroconversion could not be excluded. Figure 3 summarises the longitudinal MOG-Ab serostatus in relation to clinical attacks.

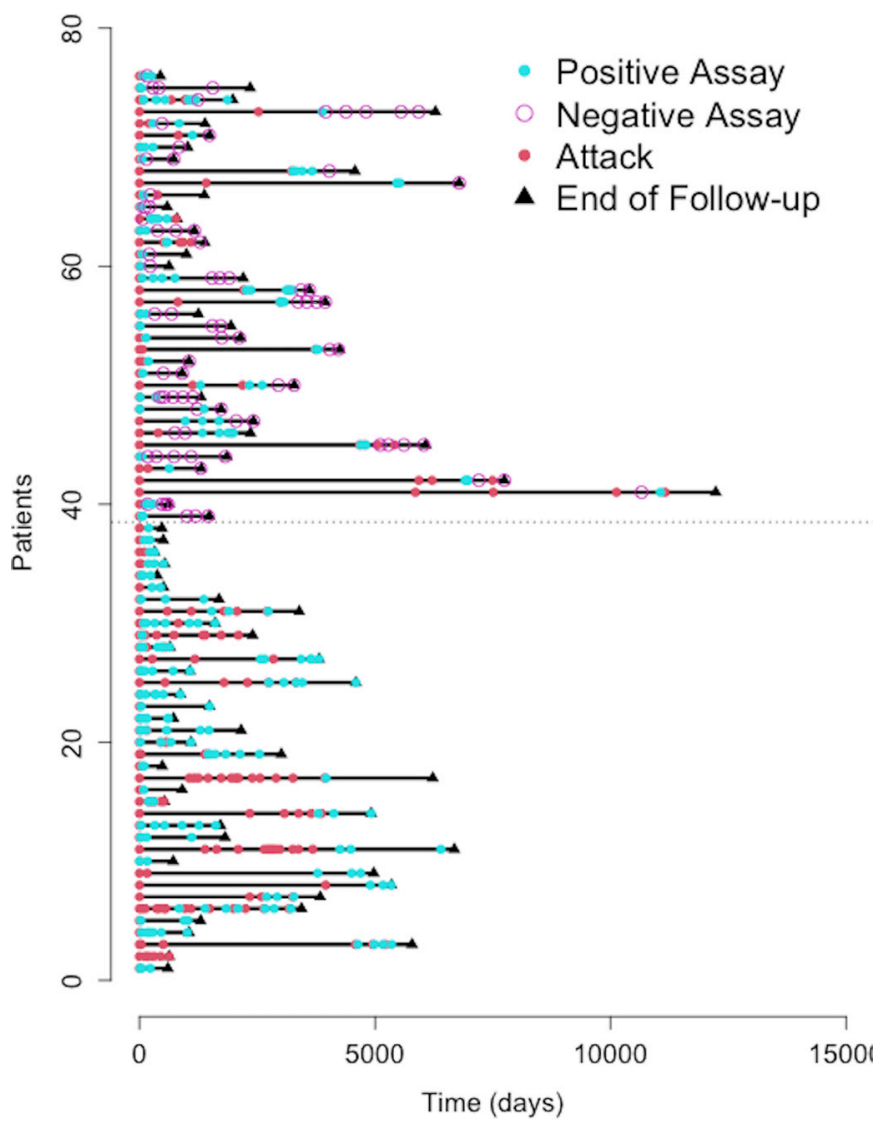

Figure 3 Clinical attacks and longitudinal MOG-Ab serostatus.

To assess the impact of MOG-Ab serostatus on clinical course we first analysed the risk of relapsing disease in those patients who became seronegative. Patients that became MOG-Ab seronegative were just as likely to have had relapsing disease as those who remained MOG-Ab positive $(45 \%$ vs $62 \%$; $p=0.229)$. To determine if longitudinal MOG-Ab serostatus influenced relapse rate we used a random effects Poisson regression model. The monthly risk of relapse was approximately $4 \%$ and reduced to $0.5 \%$ following MOG-Ab negative seroconversion (RR $0.11(0.048-0.259) ; \mathrm{p}<0.001$, online supplemental table $3)$. Figure 2 illustrates clinical course of patients in relation to longitudinal MOG-Ab serostatus. Unfortunately, it was not possible to assess the impact of MOG-Ab titre on risk of relapsing and monophasic disease as these data were not available in all patients.

In univariable analysis, patients were more likely to become MOG-Ab negative if they presented with TM $(\mathrm{p}=0.018)$, had an infective trigger $(\mathrm{p}=0.041)$, or had less three attacks $(\mathrm{p}=0.039$ ) overall (online supplemental table 1). A trend towards MOG-Ab negative seroconversion was noted with long-term IS ( $72 \%$ vs $33 \%$; $p=0.057$ ) but no specific treatment was associated with a higher likelihood of subsequent negative MOG-Ab serostatus. Longitudinal MOG-Ab serostatus was not associated with overall disability $(\mathrm{p}=0.802)$ or VA $(\mathrm{p}=0.067)$. In the multivariable analysis, TM at onset was associated with MOG-Ab negative seroconversion (OR 2.85 (1.11 to 7.30); $\mathrm{p}=0.029$ ). 


\section{DISCUSSION}

In this study that included MOGAD cases from across the UK, we found that male patients receiving $\geq 1$ month of steroid treatment at disease onset and spinal cord involvement at first presentation had a lower risk of relapsing disease. A transition to MOG-antibody negative serostatus occurred in around half of patients and was associated with a lower risk of relapse. Spinal cord involvement at onset was associated with negative MOG-Ab seroconversion.

There is wide variation in the reported rates of relapsing MOGAD in retrospective cohorts $(27 \%-80 \%) \cdot{ }^{1-5}$ Unsurprisingly, the highest proportion of relapsing disease has been observed in studies with longer follow-up duration. The stratification of relapse risk at disease onset is important when considering the long-term approach to MOGAD treatment. In this study, we analysed relapsing and monophasic patients to identify prognostic factors related to relapse and disability. We also included an incident cohort analysis to assess for observer bias.

The clinical characteristics of these patients with MOGAD were similar to previous reports, with relapsing disease observed in $55 \%$ of cases. ${ }^{1-6} 10$ A relapse rate of $18 \%$ in incident cases was lower than other reported studies $(27 \%-36 \%) \cdot{ }^{126}$ It has been shown previously that the risk of relapse is highest in the first year and in this study only cases with at least 12 months follow-up were included. ${ }^{1}$ Furthermore, the median follow-up duration of incident cases was almost 3 years though it is noteworthy that the risk of relapse in one study was $45 \%$ at 2 years and $62 \%$ at 5 years. ${ }^{2}$

We found that in male patients the time to first relapse was longer and the overall risk of relapsing disease was lower. This is similar to the findings of a recent large French study in childhood onset MOGAD. ${ }^{6}$ The explanation for this finding is uncertain, particularly as unlike other autoimmune diseases such as NMOSD, the female predominance in MOGAD is less marked. ${ }^{7}$ In our cohort patients presenting with spinal cord involvement at disease onset had a lower risk of relapsing disease and a longer latency to first relapse, reproducing findings from an Indian cohort study. ${ }^{5}$ Importantly, relapsing and monophasic patients with spinal cord involvement at disease onset were treated similarly with regards to steroid taper and had similar disease duration. As has been previously reported, a prolonged steroid taper with a first MOGAD attack was associated with a lower risk of relapsing disease. ${ }^{1510}$ In keeping with UK recommendations for MOGAD treatment, a prolonged steroid taper was more frequently observed in the incident cases. ${ }^{11}$ As mentioned previously, these cases were followed for a median of 3 years and the lower relapse rates (18\%) in this cohort may relate to the use of corticosteroids but also to disease duration. Paradoxically and in contrast to the findings by Cobo-Calvo et al, childhood onset disease was associated with relapsing disease and disability. ${ }^{6}$ This finding is explained by the preferential follow-up of children with more severe MOGAD who transition into adult neurological services. Accordingly, this parameter was excluded from the ROC analysis but a high AUC of 0.85 was maintained for predicting patients less likely to develop relapsing disease using features identifiable at first clinical presentation (male sex, spinal cord involvement, steroids $>1$ month) .

$\mathrm{VA}<6 / 36$ in at least one eye was observed in $17 \%$ of the total cohort, comparable to rates of $13 \%$ and $17 \%$ from other studies. ${ }^{16}$ In the multivariable analysis, spinal cord onset was associated with a better visual prognosis at follow-up. This relates to less optic nerve involvement in these cases but there was also a trend towards better visual outcome in patients presenting with TM with subsequent optic nerve involvement. Further exploration of this finding in a larger dataset would be of interest. Spinal cord involvement in MOGAD is frequently associated with residual bladder, bowel and erectile dysfunction and the former was present in around a third of patients in this study. ${ }^{12}$ As expected, in the univariable analysis TM was also associated with an EDSS $>3$ at long-term follow-up. Comparable to the $33 \%$ and $24 \%$ of patients presented here, $27 \%$ of a total MOGAD cohort and $22 \%$ of an incident cohort had an EDSS $>3$ in 2 large French studies. ${ }^{26}$ Several factors of interest were identified in univariable but not multivariable analyses of VA (relapsing disease, number of relapses, and a history of bilateral ON) and overall disability (number of relapses and spinal cord involvement) that could be explored further.

In this study, we were able to analyse the longitudinal profile of patients in relation to MOG-Ab serostatus. MOG-Abs became negative in $45 \%$ of cases which is higher than rates reported in other studies of MOGAD, particularly adults $(28 \%-57 \%) .{ }^{16}{ }^{13}$ This finding may relate to longer follow-up times; the median time to negative serostatus in this study was almost 3 years. Although final MOG-Ab serostatus was not associated with a relapsing disease course, longitudinal analysis of serostatus showed a reduction of $4 \%-0.5 \%$ in monthly relapse risk with MOG-Ab negative serostatus. Only 1 patient relapsed within 6 months of a negative MOG-Ab assay. These findings support the prognostic value of serial antibody testing and consideration of MOG-Ab serostatus in long term treatment decisions.

This study benefited from a nationwide catchment of patients across the UK that were followed in a single centre but is not without limitation. As with previous studies, higher relapse rates were observed in the total cohort as compared with incident cases. In particular, childhood onset patients had higher rates of disability with longer follow-up duration due to follow-up bias. Monophasic patients were followed for a median of 3 years which is longer than the median time of 15.8 months to first relapse in a nationwide French study. ${ }^{2}$ However, relapsing patients had a longer duration of follow-up as compared with monophasic cases. With a larger incident cohort, a separate analysis could have been performed to address this. However, prognostic factors related to male sex, onset attack topography, onset attack treatment and MOG-Ab serostatus were less likely to be influenced by 
these differences and are the key findings of this study. Importantly subgroup analyses were performed to assess for the impact of differences in disease duration and were factored into data interpretation. In this study we defined MOGAD on the basis of serum MOG-Abs rather than serum and CSF. Intrathecal synthesis of MOG-Ab has been reported and it would be interesting to explore this further in a prospective study that includes CSF analysis. ${ }^{14}$ In a specialised centre referral bias towards a more severe relapsing disease is also a likely factor, though similar numbers of relapsing and monophasic patients were present overall making group comparisons possible. As with all observational studies the results of the analyses do not hold the same weight as those of randomised controlled studies. In particular, for the analysis of observational datasets, the onus is on accounting for possible confounding when drawing conclusions on possible causal effects. While multivariable modelling is a powerful tool in adjudging for possible confounding, the impact of conclusions is given further weight by external validation against a new dataset and will be the focus of future research.

In summary, we have identified that male patients with spinal cord involvement at disease onset have a lower risk of relapsing disease and longer latency to first relapse. Steroid treatment for at least 1 month at disease onset was also associated with a monophasic disease course. MOG-Ab negative seroconversion was associated with a lower risk of relapse and may help inform treatment decisions and duration.

\section{Author affiliations}

${ }^{1}$ Department of Neurology, The Walton Centre NHS Foundation Trust, Liverpool, UK

${ }^{2}$ Department of Neurology, Salford Royal Hospital, Salford, UK

${ }^{3}$ Liverpool Cancer Trials Unit, University of Liverpool, Liverpool, UK

${ }^{4}$ Department of Neurology, Alder Hey Children's NHS Foundation Trust, Liverpool, UK ${ }^{5}$ Institute of Infection and Global Health, University of Liverpool, Liverpool, UK ${ }^{6}$ Nuffield Department of Clinical Neurosciences, University of Oxford, Oxford, UK ${ }^{7}$ Anne Rowling Regenerative Neurology Clinic, University of Edinburgh, Edinburgh, UK

${ }^{8}$ UK Dementia Research Institute, University of Edinburgh, Edinburgh, Scotland ${ }^{9}$ Department of Neurology, Cleveland Clinic Abu Dhabi, Abu Dhabi, UAE

Contributors Substantial contributions to the conception/design of work (SH, AJ, PW), data acquisition (SH, DW, VK, PK, SL, KMut, RK, MW, KMur, DH, PW, AJ), analysis or interpretation of data (SH, RJ, PW, AJ), drafting the work or revising it critically for important intellectual content (SH, PW, AJ), final approval of the version published (all authors). SH accepts full responsibility for the work and/ or conduct of the study, had access to the data, and controlled the decisison to publish.

Funding The authors have not declared a specific grant for this research from any funding agency in the public, commercial or not-for-profit sectors.

Map disclaimer The inclusion of any map (including the depiction of any boundaries therein), or of any geographic or locational reference, does not imply the expression of any opinion whatsoever on the part of BMJ concerning the legal status of any country, territory, jurisdiction or area or of its authorities. Any such expression remains solely that of the relevant source and is not endorsed by BMJ. Maps are provided without any warranty of any kind, either express or implied.

Competing interests None declared.

Patient consent for publication Not applicable.
Ethics approval The study was approved by the Research Ethics Service, NRES Committee London- Hampstead, Ref. no. 15/L0/1433. All patients provided written informed consent.

Provenance and peer review Not commissioned; externally peer reviewed.

Data availability statement Data are available upon reasonable request.

Supplemental material This content has been supplied by the author(s). It has not been vetted by BMJ Publishing Group Limited (BMJ) and may not have been peer-reviewed. Any opinions or recommendations discussed are solely those of the author(s) and are not endorsed by BMJ. BMJ disclaims all liability and responsibility arising from any reliance placed on the content. Where the content includes any translated material, BMJ does not warrant the accuracy and reliability of the translations (including but not limited to local regulations, clinical guidelines, terminology, drug names and drug dosages), and is not responsible for any error and/or omissions arising from translation and adaptation or otherwise.

Open access This is an open access article distributed in accordance with the Creative Commons Attribution Non Commercial (CC BY-NC 4.0) license, which permits others to distribute, remix, adapt, build upon this work non-commercially, and license their derivative works on different terms, provided the original work is properly cited, appropriate credit is given, any changes made indicated, and the use is non-commercial. See: http://creativecommons.org/licenses/by-nc/4.0/.

ORCID iD

Saif Huda http://orcid.org/0000-0003-0980-3750

\section{REFERENCES}

1 Juryńczyk M, Messina S, Woodhall MR, et al. Clinical presentation and prognosis in MOG-antibody disease: a UK study. Brain 2017;140:3128-38.

2 Cobo-Calvo A, Ruiz A, Maillart E, et al. Clinical spectrum and prognostic value of CNS MOG autoimmunity in adults: the MOGADOR study. Neurology 2018;90:e1858-69.

3 Senanayake B, Jitprapaikulsan J, Aravinthan M, et al. Seroprevalence and clinical phenotype of MOG-IgG-associated disorders in Sri Lanka. J Neurol Neurosurg Psychiatry 2019;90:jnnp2018-320243-83.

4 Jarius S, Ruprecht K, Kleiter I, et al. MOG-IgG in NMO and related disorders: a multicenter study of 50 patients. Part 2: epidemiology, clinical presentation, radiological and laboratory features, treatment responses, and long-term outcome. J Neuroinflammation 2016;13:280.

5 Pandit L, Mustafa S, Nakashima I, et al. MOG-IgG-associated disease has a stereotypical clinical course, asymptomatic visual impairment and good treatment response. Mult Scler J Exp Transl Clin 2018;4:205521731878782-9.

6 Cobo-Calvo A, Ruiz A, Rollot F, et al. Clinical features and risk of relapse in children and adults with myelin oligodendrocyte glycoprotein antibody-associated disease. Ann Neurol 2021;89:30-41.

7 Sato DK, Callegaro D, Lana-Peixoto MA, et al. Distinction between MOG antibody-positive and AQP4 antibody-positive NMO spectrum disorders. Neurology 2014;82:474-81.

8 Whittam DH, Karthikeayan V, Gibbons E, et al. Treatment of MOG antibody associated disorders: results of an international survey. $J$ Neurol 2020;267:3565-77.

9 Waters PJ, Komorowski L, Woodhall M, et al. A multicenter comparison of MOG-IgG cell-based assays. Neurology 2019;92:10.1212/WNL.0000000000007096-1255.

10 Ramanathan S, Mohammad S, Tantsis E, et al. Clinical course, therapeutic responses and outcomes in relapsing MOG antibodyassociated demyelination. J Neurol Neurosurg Psychiatry 2018;89:127-37.

11 Juryńczyk M, Jacob A, Fujihara K, et al. Myelin oligodendrocyte glycoprotein (MOG) antibody-associated disease: practical considerations. Pract Neurol 2019;19:187-95.

12 Mariano R, Messina S, Roca-Fernandez A, et al. Quantitative spinal cord MRI in MOG-antibody disease, neuromyelitis optica and multiple sclerosis. Brain 2021;144:198-212.

13 Waters P, Fadda G, Woodhall M, et al. Serial anti-myelin oligodendrocyte glycoprotein antibody analyses and outcomes in children with demyelinating syndromes. JAMA Neurol 2020;77:82-12.

14 Akaishi T, Takahashi T, Misu T, et al. Difference in the source of Anti-AQP4-IgG and Anti-MOG-IgG antibodies in CSF in patients with neuromyelitis optica spectrum disorder. Neurology 2021;97:e1-12. 